\title{
A New Low Complexity NLOS Identification Approach Based on Maximum Slope and Skewness of Energy Block for 60GHz System
}

\author{
Xiaolin Liang ${ }^{1}$, Hao Zhang ${ }^{1,2}$, Tingting $\mathrm{Lv}^{1}$ and T. Aaron. Gulliver ${ }^{2}$ \\ ${ }^{1}$ College of Information Science and Engineering, Ocean University of China, \\ Qingdao, 266100, China \\ ${ }^{2}$ Department of Electrical and Computer Engineering, University of Victoria, \\ Victoria V8W 3P6, Canada \\ 1xiaolin87liang@163.com, ${ }^{2}$ zhanghao@ouc.edu.cn \\ ${ }^{3}$ lvtingting33@163.com, ${ }^{4}$ agullive@ece.uvic.ca
}

\begin{abstract}
The major problem of indoor localization is the presence of non-line-of-sight (NLOS) channels. In order to perform the NLOS identification, in this paper, we propose a novel NLOS identification technique based on the ratio values of the maximum slope and skewness of energy block of the received signal using energy detector. In particular, the IEEE 802.15.3c $60 \mathrm{GHz}$ channel models are used as examples and the above statistics is found to be explained in detail. The simplicity of the proposed approach lies in the use of the parameters of the energy-based time of arrival (TOA) Estimation algorithm. The CM1 (LOS) and CM2 (NLOS) channel models of the standard IEEE 802.15.3c channel models are used. Numerical simulations results show that the correct identification of channel models with the proposed approach is better than with the multipath channel statistics based approach.
\end{abstract}

Keywords: NLOS identification, maximum slope, skewness, 60GHz, Energy Detector, IEEE 802.15.3c

\section{Introduction}

The location of a mobile terminal (MT) can be estimated using different parameters of a received signal, such as the time-of-arrival (TOA), angle-of-arrival (AOA), and/or the received signal strength (RSS). Impulse Radio (IR) $-60 \mathrm{GHz}$ has a great potential for accurate ranging and localization systems due to its very wide bandwidth and capability in resolving individual multipath components [1-6]. Therefore, the TOA of the received signal can be estimated with high accuracy for the $60 \mathrm{GHz}$ systems if the first arriving path has been identified precisely. One of the major challenges for localization systems is the mitigation of non-line-of-sight (NLOS) effects. If the direct path between a fixed terminal (FT) (An FT is usually a base station in a cellular network or an anchor node in a sensor network.) and the MT is being obstructed, the TOA of the signal to the FT will be delayed, which introduces a positive bias. Using such NLOS TOA estimates during the localization of the MT position may significantly degrade the positioning accuracy. Hence, FTs that are under the NLOS condition have to be identified and their effects have to be mitigated.

The NLOS identification and mitigation techniques have been discussed extensively in the literature, but mainly within the cellular network framework [7-13]. For example, in [7], skewness of the ranging measurements is compared with the threshold for NLOS identification, where the measurement noise variance is assumed to be known. In [8], a decision-theoretic NLOS identification framework is presented, where various hypothesis tests are discussed for known and unknown probability density functions (PDFs) of the 
TOA measurements. Guvenc et. al., [9-13] used mean excess delay, root mean square (RMS) delay, and kurtosis of multipath channel as NLOS identification metrics. When the statistics of kurtosis, mean excess, and RMS delays are priori known, likelihood ratio tests can be performed for hypothesis selection. Another method was proposed by Heidari et. $a l$., [10]. They tried to find the first detected path of the received signal as a peak of the filtered Channel Impulse Radio. As similar to the previous method, this technique also used joint likelihood function using the mean excess delay $\tau_{\text {med }}$, total received power $P_{t o t}$ and the hybrid of the power of the first detected path and TOA of the first detected path $\xi_{h y b}$.Venkatesh et. al., [11] identified the channel based on TOA, RSS, and RMS delay spread (RDS) of the received signal. The conditional distributions of the TOA, RSS, and RDS estimates are functions of the distance and the channel state. Provided that the physical distance between the transmitted and receive nodes is known exactly, the state of the channel can be identified by comparing the likelihood values for each of the estimates (TOA, RSS, and RDS), conditioned on the distance. Shimizu et. al., [12] performed intensive measurements of path-loss and delay-profile characteristics of LOS and NLOS environments in a suburban residential area. Based on their analysis, they found that the delay spread was dependent on distance, and the NLOS delay spread was found to be several times larger than that of the LOS case. The skewness of delay spread for the NLOS cases ranged from 80 to $200 \mathrm{~ns}$, which was an order of magnitude larger than that of the LOS case.

In this paper, we propose a new LOS/NLOS identification approach for the IR -60 $\mathrm{GHz}$ signal, which is based on the maximum slope and skewness of energy block of the received signal using energy detector. Firstly, we use the energy detector TOA estimation algorithm for the estimation the TOA. Secondly, we characterize the maximum slope and skewness of the energy block of the received signal. Finally, we use a threshold test for LOS/NLOS identification.

The remainder of this paper is organized as follows. Section 2 describes the signal and channel model. Section 3 describes LOS/NLOS identification approach and Section 4 presents the results of the numerical simulations. The concluding remarks are given in Section 5.

\section{System Model}

Currently, there are two important standards that have been developed for the $60 \mathrm{GHz}$ wireless communications systems, IEEE 802.15.3c and IEEE 802.11ad [14-15]. In this paper, the channel models in IEEE 802.15. 3c standard are used because it is specifically designed for Wireless Personal Area Networks (WPAN) and thus encompasses typical indoor environments. Further, these are the most widely employed models for the $60 \mathrm{GHz}$ systems. The IEEE 802.15.3c standard was the first developed for high data rate short-range wireless systems. The physical layer was designed to support the transmission of data within a few meters at a maximum data rate of $2 \mathrm{Gbps}$. These models have been developed for communications in the frequency band 57 to $66 \mathrm{GHz}$ in indoor residential, indoor office and library environments (with differences largely due to the LOS and NLOS characteristics) [16-20]. In this paper, a Pulse Position Modulation Time Hopping (PPM-TH) $60 \mathrm{GHz}$ signal is employed for ranging purposes. The propagation delay $\hat{\tau}$, between the transmitter and receiver is estimated for use in localization.

\subsection{GHz Signal}

The PPM-TH $60 \mathrm{GHz}$ signals have a very short duration (typically 100 picoseconds or less), and can be expressed as: 
$s(t)=\sum_{-\infty}^{\infty} p\left(t-j T_{s}-C_{j} T_{c}-a_{j} \varepsilon\right)$

where $T_{s}$ is the symbol time. The Time Hopping $(\mathrm{TH})$ code represented by $C j$ is a pseudorandom integer-valued sequence which is unique for each user to limit multiple access interference, and $T_{c}$ is the chip time. The PPM time shift is $\varepsilon$ so that if $a_{j}$ is 1 , the signal is shifted in time by $\varepsilon$, while if $a_{j}$ is 0 , there is no shift. Many pulse shapes have been proposed for the $60 \mathrm{GHz}$ systems. In this paper a Gaussian pulse is employed which is multiplied by the carrier signal to give:

$p(t)=\frac{\sqrt{2}}{\alpha} \exp \left(-2 \pi \frac{t^{2}}{\alpha^{2}}\right) \cos \left(2 \pi f_{c} t\right)$

where $\alpha$ is the shape factor and $f_{c}$ is the carrier frequency which here is $f_{c}=60 \mathrm{GHz}$. A smaller shape factor results in a shorter duration pulse and a larger bandwidth.

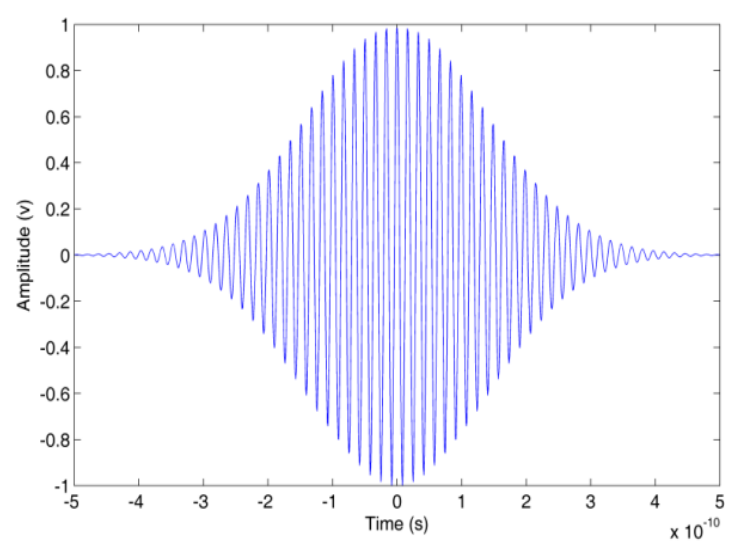

Figure 1. Waveform of $60 \mathrm{GHz}$ Signal

\subsection{Signal Shift and Path Loss}

The path loss is defined as the ratio of the received signal power to the transmit signal power and it is very important for link budget analysis. Unlike narrow-band system, the path loss for a wide-band system such as mm-wave system is both distance and frequency dependent. In order to simplify the models, it is assumed that the frequency dependence Path Loss is negligible and only distance dependence path loss is modeled. The signal path loss, which depends on the propagation distance and the channel (IEEE 802.15.3c), is described by:

$P L(d)[d B]=P L_{0}+10 \cdot n \log _{10}\left(\frac{d}{d_{0}}\right)+X_{\sigma}[d B] ; d \geq d_{0}$

where $d_{0}$ and $d$ denote the reference distance, and distance respectively. $n$ is the path loss exponent, $X_{\sigma}$ is that the unit $\mathrm{dB}$, with mean zero and variance $\sigma_{s}$ for a Gaussian random variable. Table 1 , summarizes the values of $\mathrm{n}, P L_{0}, \sigma_{s}$ for different environments and scenarios. 
Table 1. Values of $\boldsymbol{n}, P L_{0}, \sigma_{s}$ for Different Environments and Scenarios

\begin{tabular}{cccc}
\hline environments & $n$ & $P L_{0}$ & $\sigma_{s}$ \\
\hline indoor residential (LOS) & 1.53 & 75.1 & 1.50 \\
indoor residential (NLOS) & 2.44 & 86.0 & 6.20 \\
indoor office (LOS) & 1.16 & 84.6 & 5.40 \\
indoor office (NLOS) & 3.74 & 56.1 & 8.60 \\
\hline
\end{tabular}

The signal shift can be expressed as:

$t=d t *$ floor $((d / c) / d t)$

where $d$ denotes the distance between the transmitter and receiver, $d t$ is the sampling period and $c$ is the speed of light which is $299792458 \mathrm{~m} / \mathrm{s}$ in the air.

\subsection{Multipath Fading Channel}

The received signal can be written as:

$r(t)=\sum_{n=1}^{N} \alpha_{n} p\left(t-\tau_{n}\right)+n(t)$

where $N$ is the number of received multipath components, $\alpha_{n}$ and $\tau_{n}$ denotes the amplitude and delay of the nth path respectively, $p(t)$ is the received $60 \mathrm{GHz}$ pulse and $n(t)$ is Additive White Gaussian Noise (AWGN) with zero mean and two sided power spectral density $\mathrm{N}_{0} / 2$. Equation (5) can be rewritten as:

$r(t)=s(t) * h(t)+n(t)$

where $s(t)$ is the transmitted signal, and $h(t)$ is the channel impulse response which can be expressed as:

$h(t, \theta)=\sum_{k=1}^{K} \sum_{l=1}^{L_{k}} \mu_{k l} \delta\left(t-T_{k}-\tau_{k l}\right) \delta\left(\theta-\theta_{k}-\omega_{k l}\right)$

where $\delta($.$) is the dirac delta function, K$ is the number of clusters, $L_{k}$ is the number of rays in the $k^{\text {th }}$ cluster, and $\mu_{k l}, \tau_{k l}$ and $\omega_{k l}$ denote the complex amplitude, delay and azimuth of the $k^{\text {th }}$ ray of the $l^{t^{h}}$ cluster, respectively. Similarly, $T_{k}$ and $\theta_{k}$ represent the delay and mean Angle of Arrival (AOA) of the $k^{\text {th }}$ cluster.

\subsection{Energy Detector}

After the amplifier, the received signals are squared, and then input to an integrator with integration period $T b$. Because of the inter-frame leakage due to multipath signals, the integration duration is $3 T_{f} / 2$, so the number of signal values for energy detector is $N=$ $3 T_{f} / 2 T b$. The integrator outputs can be expressed as:

$z[n]=\sum_{i=1}^{N} \int_{(i-1) T_{f}+\left(c_{j}+n-1\right) T b}^{(i-1) T_{f}+\left(c_{j}+n\right) T b} r^{2}(t) d t$

where $n \in\{1,2, . ., N\}$ denotes the sample index with respect to the starting point of the integration period and $N$ is the number of pulses per symbol. Here, $N$ is set to 1 , so the integrator outputs are: 
$z[n]=\sum_{i=1}^{N} \int_{\left(c_{j}+n-1\right) T b}^{\left(c_{j}+n\right) T b} r^{2}(t) d t$

If $z[n]$ is the integration of noise only, it has a centralized Chi-square distribution, while it has a non-centralized Chi-square distribution if a signal is present. The mean and variance of the noise and signal values are given by [17] respectively.

$$
\begin{aligned}
& \mu_{0}=F \sigma^{2}, \sigma_{0}=2 F \sigma^{4} \\
& \mu_{e}=F \sigma^{2}+E_{n}, \sigma_{e}^{2}=2 F \sigma^{4}+4 \sigma^{2} E_{n}
\end{aligned}
$$

where $E_{n}$ is the signal energy within the $n^{\text {th }}$ integration period and $F$ is the number of degrees of freedom given by $F=2 B T b+1$. Here $B$ is the signal bandwidth.

\section{LOS/NLOS Identification}

\subsection{Description of the Parameters}

In this paper, we distinguish between the LOS or NLOS scenarios by exploiting the statistics of the received signal by energy detector. The maximum slope and skewness of the energy block is used in order to identify the LOS and NLOS scenarios respectively. The slope of the energy values is considered as a measure. These values are divided into $(N-M+1)$ groups, with $M$ values in each group. The slope for each group is calculated using a least squares line-fit. The maximum slope can then be expressed as:

$$
M S=\max _{1 \leq n \leq N-M+1} \text { slope }\{\text { linefit }(z[n], z[n+1], \ldots \ldots, z[n+M-1])\}
$$

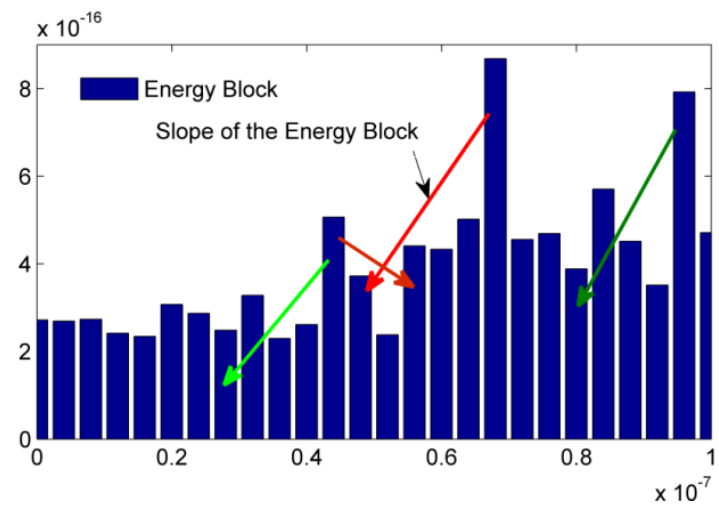

Figure 2. Slope of the Energy Block

The skewness is given by:

$$
S=\frac{1}{(N-1) \delta^{3}} \sum_{i=1}^{N}\left(x_{i}-\mu_{x}\right)^{3}
$$

where $\mu_{x}$ is the mean value, and $\delta$ is the standard deviation of the energy values. The skewness for a normal distribution is 0 ; in fact any symmetric data will have a skewness of zero. Negative values of skewness indicate that the data is skewed left, while positive values indicate data that is skewed right. Skewed left indicates that the left tail is long relative to the right tail, while skewed right indicates the opposite. For noise only (or very low SNR), and sufficiently large $F, S \approx 0$. As the SNR increases, $S$ will tend to increase. 


\subsection{Identification Approach}

The ratio values of slope and skewness of the energy block can be obtained for both LOS and NLOS scenarios using sample channel realizations from both scenarios. Here, we used sample channel realizations of the IEEE 802.15.3c standard channel models in order to obtain the values of the slope of the energy slope for both LOS and NLOS.

In order to examine the characteristics of the ratio of maximum slope and skewness, the CM1.1 (residential LOS) and CM2.1 (residential NLOS) channel models from the IEEE 802.15.3c standard are employed. For each SNR value, 1000 channel realizations are generated and sampled at $f_{c}=1 \cdot e^{10} \mathrm{~Hz}$. The other system parameters are $T_{f}=200 \mathrm{~ns}$, $T_{c}=1 n s$, the value of $T b$ is from $1 n s$ to $4 n s$ and $N=1$. Each realization has a TOA uniformly distributed within $\left(0-T_{f}\right)$. Here the SNR ranges from $1 \mathrm{~dB}$ to $9 \mathrm{~dB}, 1000$ channel realizations are generated for each SNR. The CM1.1 (residential LOS) and CM2.1 (residential NLOS) channel models from the IEEE802.15.3c standard are employed. Here are $9 * 1000$ samples which are got for each channel model. The relationship between maximum slope and SNR are shown in Figures 3-6.

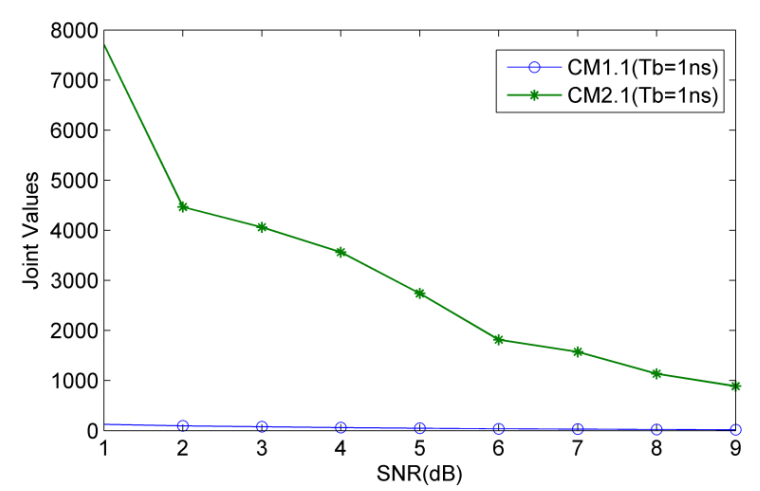

Figure 3. Joint Values with Respect to SNR (CM1.1 and CM2.1 with Tb=1ns)

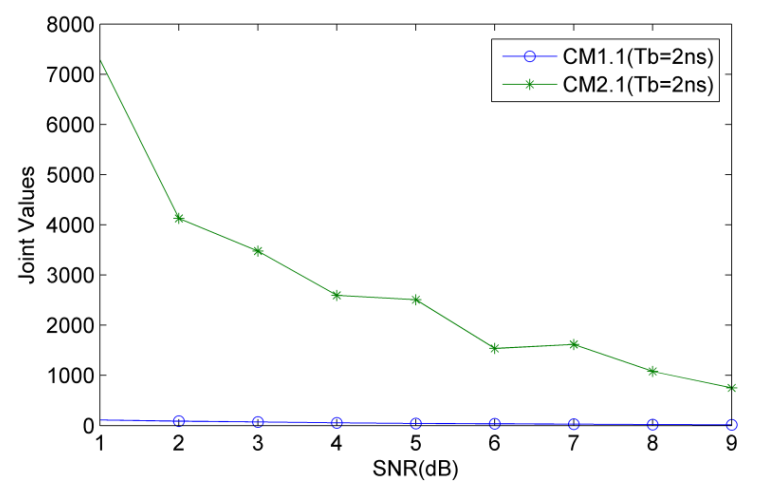

Figure 4. Joint Values with Respect to SNR (CM1.1 and CM2.1 with Tb=2ns) 


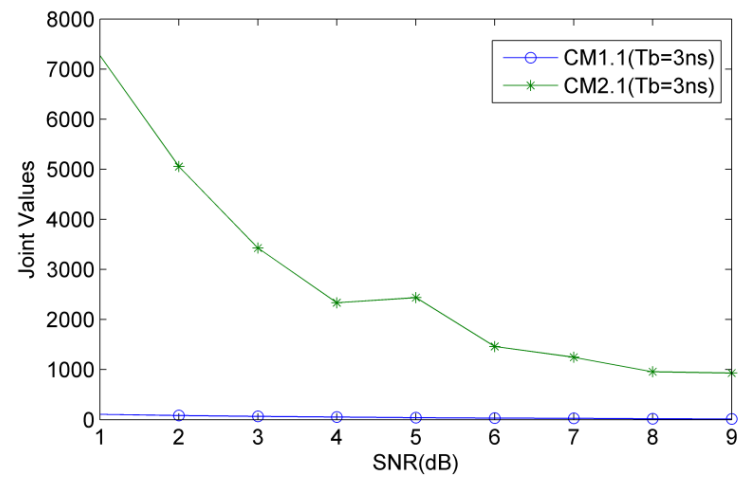

Figure 5. Joint Values with Respect to SNR (CM1.1 and CM2.1 with Tb=3ns)

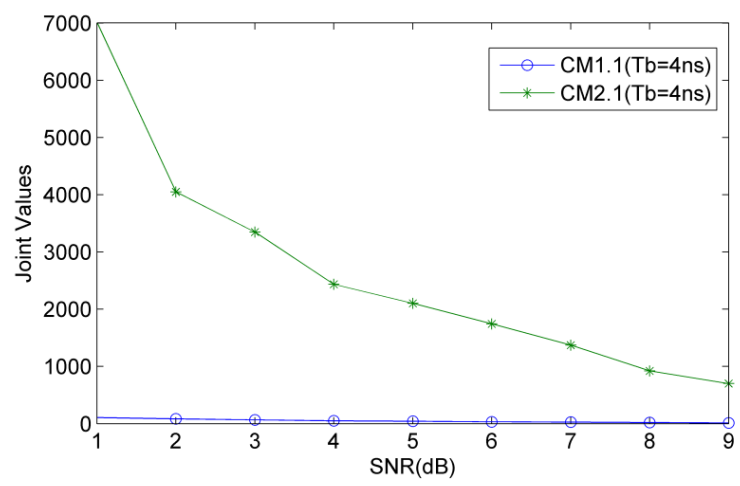

Figure 6. Joint Values with Respect to SNR (CM1.1 and CM2.1 with Tb=4ns)

From Figures 3-6, results show that the multiplied values of maximum slope and skewness is monotonous with respect to SNR (both LOS and NLOS environment) in the $60 \mathrm{GHz}$ wireless communication system respectively. But we find maximum of joint values (LOS) is even less than minimum of joint values (NLOS) when TX is 360, minimum of joint values (LOS) is even larger than maximum of joint values (NLOS) when TX is others. So we propose a novel method to identify the LOS and NLOS which can be expressed as:

$\Theta=\alpha\left\{\begin{array}{l}\left\{\begin{array}{l}>\alpha_{M S} \Rightarrow N L O S \\ <\alpha_{M S} \Rightarrow L O S\end{array} \quad \text { TX }=360^{\circ}\right. \\ \left\{\begin{array}{l}>\alpha_{M S} \Rightarrow L O S \\ <\alpha_{M S} \Rightarrow N L O S\end{array} \quad \text { others }\right.\end{array}\right.$

where $\alpha_{M S}$ is threshold which is chosen to identify LOS and NLOS, TX, $R X$ is beam-width of measured transmitter and receiver antenna respectively. Aiming at ranges $[\mathrm{A}, \mathrm{B}] \mathrm{dB}$ for SNR, $\alpha_{M S}$ can be shown as follows :

$\alpha_{M S}=\min \left\{\begin{array}{l}\operatorname{mean}\left[\operatorname{find}\left(P\left(\operatorname{abs}(M S)<\left.\Theta_{1}\right|_{A}\right) \geq \Phi\right)\right] \\ \operatorname{mean}\left[\operatorname{find}\left(P\left(\operatorname{abs}(M S)<\left.\Theta_{2}\right|_{B}\right) \geq \Phi\right)\right]\end{array}\right\}$

where $\Theta_{1}$ and $\Theta_{2}$ are threshold which meet the condition $P(M S>\Theta) \geq \Phi$ where SNR is upper and lower limits. $\Phi$ is probability value which is required for choosing suitable threshold for NLOS identification. 


\section{Simulated Results}

In order to verify the effectiveness and practicality of the algorithm, so we make a lot of simulations using IEEE 802.15.3c channel models. The CM1.2, CM1.3, CM1.4 (residential LOS) and CM2.2, CM2.3, CM2.4 (residential NLOS) channel models from the IEEE802.15.3c standard are employed. For each SNR value, 1000 channel realizations are generated and sampled at $f_{c}=1 \cdot e^{10} \mathrm{~Hz}$. The other system parameters are $T_{f}=200 \mathrm{~ns}$, $T_{c}=1 n s$, the value of $T b$ is from $1 \mathrm{~ns}$ to $4 \mathrm{~ns}$ and $\mathrm{N}=1$. Each realization has a TOA uniformly distributed within $\left(0-T_{f}\right)$. Here the SNR ranges from $1 \mathrm{~dB}$ to $9 \mathrm{~dB}, 1000$ channel realizations are generated for each SNR. The CM1.2, CM1.3, CM1.4 (residential LOS) and CM2.2, CM2.3, CM2.4 (residential NLOS) channel models from the IEEE802.15.3c standard are employed. Here are $9 * 1000$ samples which are got for each channel model. The relationship between joint parameter and SNR are shown in Figures 7-18.

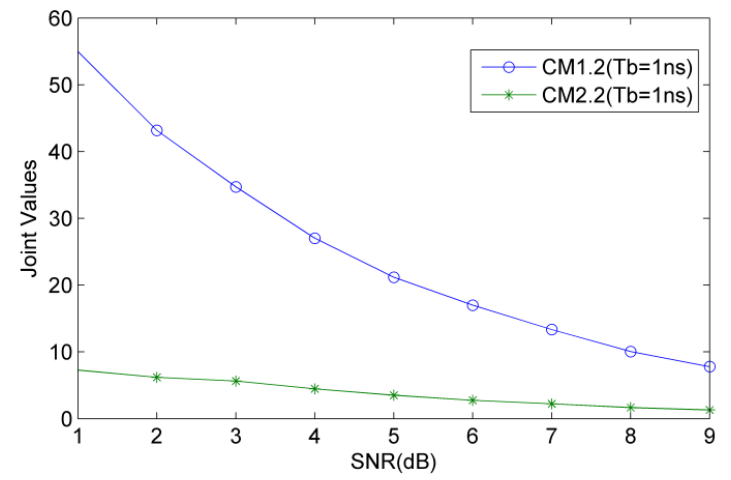

Figure 7. Joint Values with Respect to SNR (CM1.2 and CM2.2 with Tb=1ns)

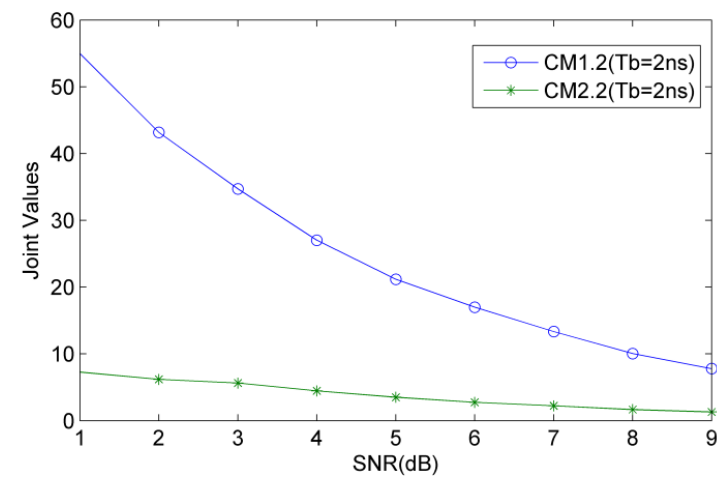

Figure 8. Joint Values with Respect to SNR (CM1.2 and CM2.2 with Tb=2ns)

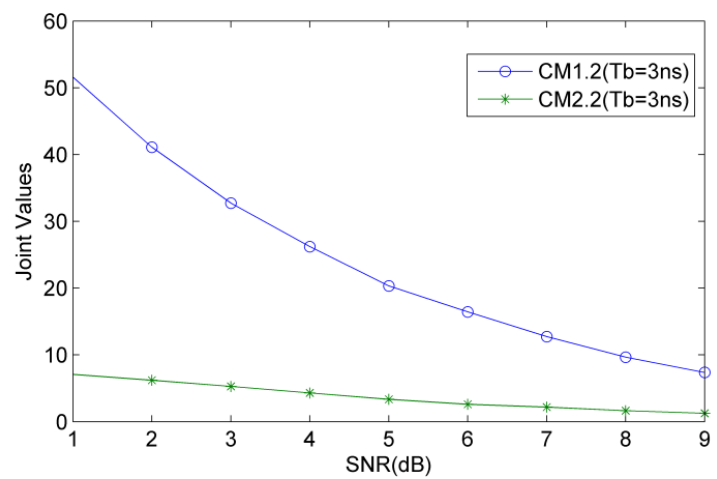

Figure 9. Joint Values with Respect to SNR (CM1.2 and CM2.2 with Tb=3ns) 


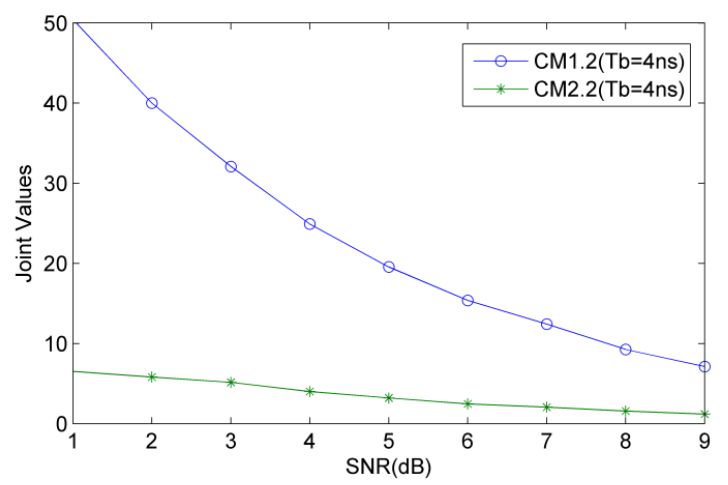

Figure 10. Joint Values with Respect to SNR (CM1.2 and CM2.2 with Tb=4ns)

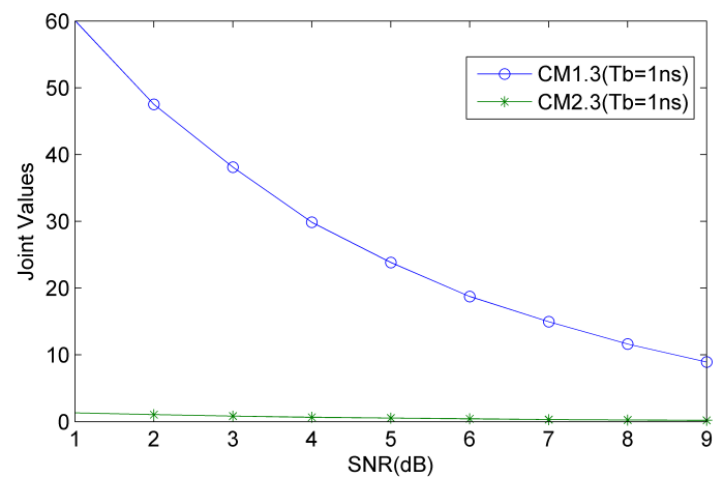

Figure 11. Joint Values with Respect to SNR (CM1.3 and CM2.3 with Tb=1ns)

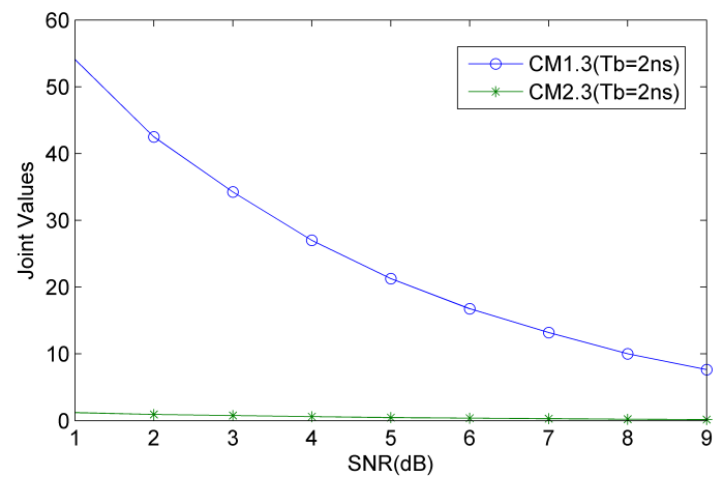

Figure 12. Joint Values with Respect to SNR (CM1.3 and CM2.3 with Tb=2ns)

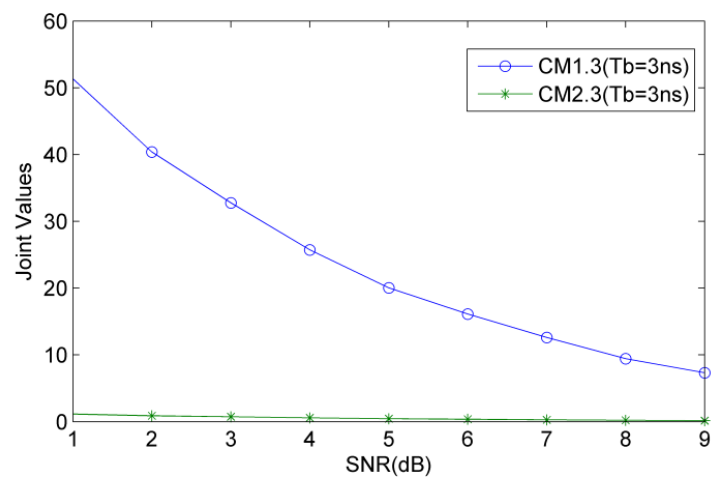

Figure 13. Joint Values with Respect to SNR (CM1.3 and CM2.3 with Tb=3ns) 


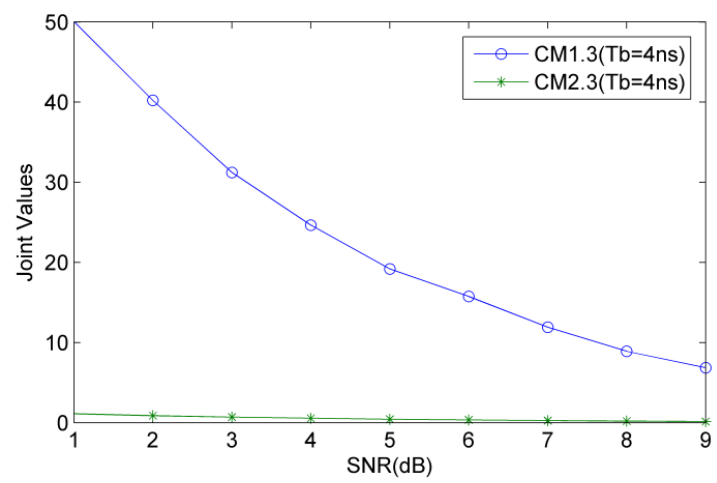

Figure 14. Joint Values with Respect to SNR (CM1.3 and CM2.3 with Tb=4ns)

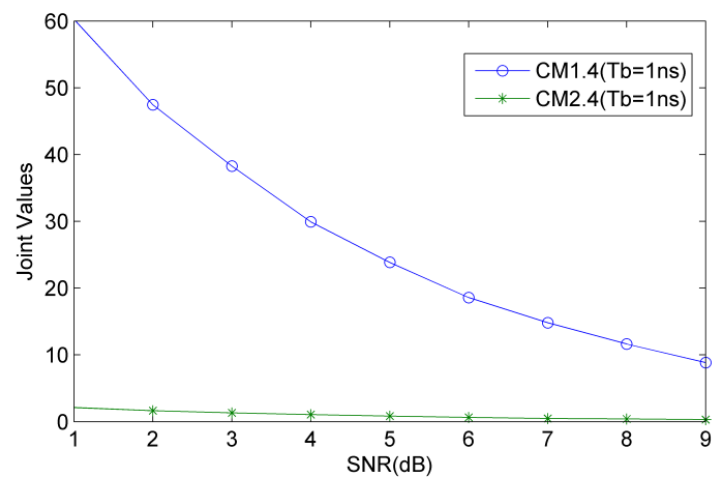

Figure 15. Joint Values with Respect to SNR (CM1.4 and CM2.4 with Tb=1ns)

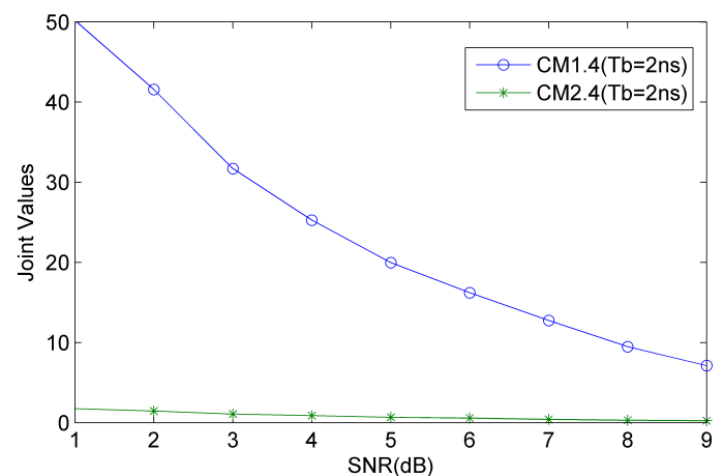

Figure 16. Joint Values with Respect to SNR (CM1.4 and CM2.4 with Tb=2ns)

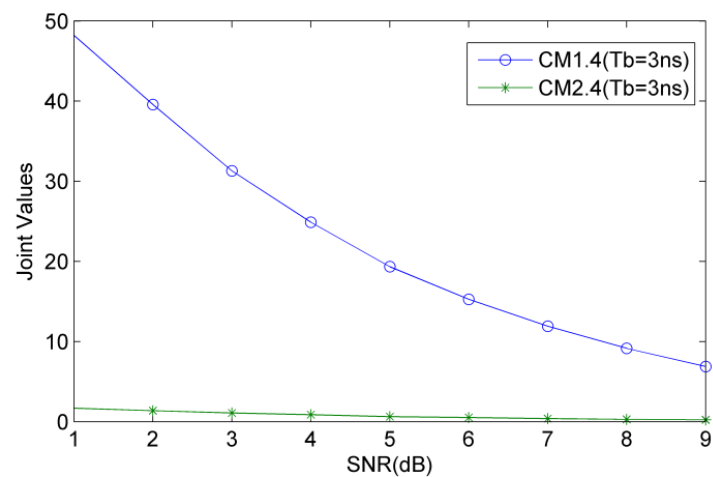

Figure 17. Joint Values with Respect to SNR (CM1.4 and CM2.4 with Tb=3ns) 


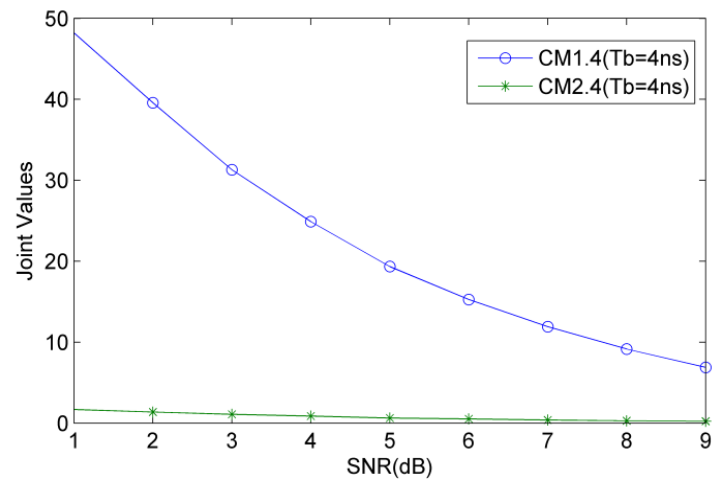

Figure 18. Joint Values with Respect to SNR (CM1.4 and CM2.4 with Tb=4ns)

From Figures 7-18, results show that joint parameter can identify the LOS and NLOS environments so long as the threshold $\alpha_{M S}$ can be fixed better.

\section{Conclusion}

In this paper, we presented a novel approach to deal with non-line-of-sight propagation that relies solely on features extracted from the received waveform. This technique does not require formulation of explicit statistical models for the features which is based on the maximum slope and skewness of energy block of the received signal using energy detector. In order to verify the effectiveness and practicality of the algorithm, so we make a lot of simulations using IEEE 802.15.3c channel models. The CM1.1, CM1.2, CM1.3, CM1.4 (residential LOS) and CM2.1, CM2.2, CM2.3, CM2.4 (residential NLOS) channel models from the IEEE802.15.3c standard are employed. Results show that the joint parameter can identify the LOS and NLOS environments so long as the threshold $\alpha_{M S}$ can be fixed bitterly. We developed techniques that are capable of distinguishing LOS/NLOS propagation in NLOS conditions. Our results revealed that the proposed technique outperforms previous parametric techniques from the literature. But here is a question that the method we proposed can't identify the LOS and NLOS environments in the office. So in the future, this will be the problem which is eager to be solved for us.

\section{Acknowledgments}

The authors would like to thank colleagues from the UWB Laboratory in the College of Information Science and Engineering, Ocean University of China, for help with obtaining the measurement data. This work was supported by the Nature Science Foundation of China under Grant No. 60902005, the Qingdao International Science and Technology Cooperation Projects of Qingdao under Grant No. 12-1-4-137-hz, and the Qingdao Transformation of Scientific and Technological Achievements Guiding Plan (youth special program) under Grant No. 14-2-4-37-jch.

\section{Reference}

[1] L. Zhang, "A fully integrated $60 \mathrm{GHz}$ four channel CMOS receiver with $7 \mathrm{GHz}$ ultra-wide band width for IEEE 802.11ad standard", Communication, China, vol. 11, no. 6, (2014), pp. 42-50.

[2] S. K. Yong and C. C. Chong, "An overview of multi gigabit wireless through millimeter Wave Strategy: Potentials and Technical Challenges", EURASIP J. Wireless Communications and Networking, vol. 2007, no. 1, (2007), pp. 1-10.

[3] R. C. Daniels and R. W. Heath, "60 GHz wireless communications: emerging requirements and design recommendations”, IEEE Vehicular Strategy Society, vol. 2, (2007), pp. 41-50.

[4] C. C. Chong, F. M. Peter and Smulders, "60GHz-Millimeter-Wave Radio Principle, Strategy, and News Results", EURASIP Journal on Wireless Communications and Networking, vol. 2007, no. 1, (2007), pp. $1-8$. 
[5] S. K. Yong, P. F. Xia and V. G. Alberto, "60-GHz Strategy for Gbps WLAN and WPAN: From Theory to Practice", Beijing: Press of China Machine, (2013).

[6] R. C. Daniels and R. W. Health, " $60 \mathrm{GHz}$ wireless communications: emerging requirements and design recommendations", IEEE Vehicular Strategy Magazine, vol. 2, no. 3, (2007), pp. 41-50.

[7] M. P. Wylie and J. Holtzman, "The non-line of sight problem in mobile location estimation", in Proceedings of the 5th IEEE International Conference on Universal Personal Communications (ICUPC '96), Cambridge, Mass,USA, vol. 2, (1996) September-October, pp. 827-831.

[8] J. Borras, P. Hatrack and N. B. Mandayam, "Decision theoretic framework for NLOS identification", in Proceedings of the 48th IEEE Vehicular Technology Conference (VTC '98), Ottawa, Canada, vol. 2, (1998) May, pp. 1583-1587.

[9] I. Guvenc, C. C. Chong and F. Watanabe, "NLOS identification and mitigation for UWB localization systems", Wireless Communications and Networking Conference, 2007. WCNC, IEEE, (2007), pp. $1571-1576$

[10] M. Heidari, F. O. Akgul and K. Pahlavan, "Identification of the absence of direct path in indoor localization systems", IEEE International Symposium on Personal, Indoor, and Mobile Radio Communications, (2007), pp. 1-6.

[11] S. Venkatesh and R. M. Buehrer, "Non-line-of-sight identification in ultra-wideband systems based on received signal statistic", Microwaves, Antennas \& Propagation, IET, vol. 1, no. 6, (2007), pp. $1120-1130$.

[12] H. Shimizu, H. Masui, M. Ishii and K. Sakawa (2000) LOS and NLOS path-loss and delay characteristics at $3.35 \mathrm{GHz}$ in a residential environment. Antennas and Propagation Society International Symposium, IEEE, vol. 2, (2000), pp. 1142-1145

[13] I. Güvenç, C. C. Chong, F. Watanabe and H. Inamura, "NLOS identification and weighted least-squares localization for UWB systems using multipath channel statistics", EURASIP Journal on Advances in Signal Processing, (2008), Article ID 271984.

[14] IEEE Standard for Information strategy--Local and metropolitan area networks--Specific requirements--Part 15.3: Wireless Medium Access Control (MAC) and Physical Layer (PHY) Specifications for High Rate Wireless Personal Area Networks (WPAN) amendment 2: millimeter-wave-based alternative physical layer extension". IEEE Computer Society, IEEE 802.15.06-0474-00-003c. New York, USA, (2009).

[15] 802.11n-2009-IEEE Standard for Information strategy-- Local and metropolitan area networks--Specific requirements--Part 11: Wireless LAN Medium Access Control (MAC) and Physical Layer (PHY) Specifications Amendment 5: Enhancements for Higher Throughput”, IEEE Computer Society, IEEE 978-0-7381-6731-2. New York, USA, (2009).

[16] C. R. Andersonn and T. S. Rappaport, "In-building wideband partition loss measurements at 2.5 and 60GHz", IEEE Transactions on Wireless Communications, vol. 3, no. 3, (2004), pp. 922-928.

[17] S. Collong, G. Zaharia and G. E. Zein, "Influence of the human activity on wide-band characteristics of the $60 \mathrm{GHz}$ indoor radio channel", IEEE Transactions on Wireless Communications, vol. 3, no. 6, (2005), pp. 2396-2406

[18] A. Maltsev, R. Maslennikov and A. Sevastyanov, "Experimental investigations of 60GHz WLAN systems in office environment", IEEE Journal on Selected Areas in Communications, vol. 27, no. 8, (2009), pp. 1488-1499.

[19] M. G. Sanchez, A. V. Alejos and I. Cuinas, "Comparision of space diversity performance in indoor radio channels at $40 \mathrm{GHz}$ and $60 \mathrm{GHz}$ ", Proc. of European Conference on Wireless Strategy, Amsterdam, (2008).

[20] H. B. Yang, "Channel characteristics and transmission performance for various channel configurations at 60GHz", EURASIP Journal on Wireless Communications and Networking, vol. 2007, no. 1, (2007), pp. 43-43.

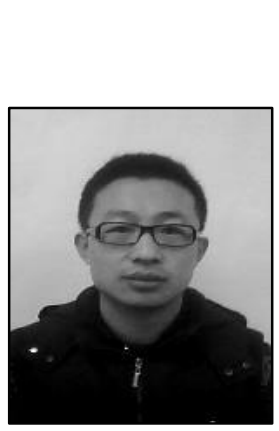

\section{Authors}

Xiaolin Liang, now studies in College of Information Science and Engineering and is a $\mathrm{Ph}$. D. candidate in Ocean University of China. His research interests include ultra-wideband radio systems, $60 \mathrm{GHz}$ wireless communication system. 


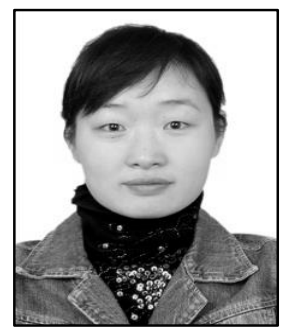

Tingting $\mathbf{L v}$, received the $\mathrm{Ph}$. D. degree in College of Information Science and Engineering from Ocean University of China in 2013. She is now a lecture in College of Information Science and Engineering. Her research interests include ultra-wideband radio systems, $60 \mathrm{GHz}$ wireless communication system.

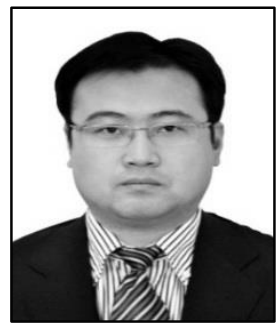

Hao Zhang, received the MBA degree in New York Institute of Technology, American in 2001 and the Ph. D. degree in Electrical and Computer Engineering from the University of Victoria, Canada in 2004. He was a Project Manager for Microsoft Inc. in United States during January 2000-May 2000. During 2004-2008, he was the Vice President for the United States Gamma Capital Inc. He is now an Adjunct Assistant Professor in the Department of Electrical and Computer Engineering. Also he becomes a professor and the $\mathrm{Ph}$. D. supervisor in College of Information Science and Engineering from Ocean University of China in 2006. His research concerns ultra-wideband radio systems, $60 \mathrm{GHz}$ wireless communication system and MIMO wireless communication.

T. Aaron. Gulliver received the $\mathrm{Ph}$. D. degree in Electrical and Computer Engineering from the University of Victoria, Canada in 1989. He is now a professor and the Ph. D. supervisor in the Department of Electrical and Computer Engineering. In 2002, he becomes a Fellow of the Engineering Institute of Canada, and in 2012 a Fellow of the Canadian Academy of Engineering. He is also a senior member of IEEE. His research concerns information theory and communication theory, algebraic coding theory and smart grid and ultra-wideband communication. 
International Journal of Signal Processing, Image Processing and Pattern Recognition Vol. 9, No. 9, (2016) 